\title{
Proximal Resection Margin
}

National Cancer Institute

\section{Source}

National Cancer Institute. Proximal Resection Margin. NCI Thesaurus. Code C138063.

The surgical margin resulting from resection of a tumor with respect to the proximal border of the specimen, as it was oriented prior to excision. 